\title{
Reference Range Comparison
}

National Cancer Institute

\section{Source}

National Cancer Institute. Reference Range Comparison. NCI Thesaurus. Code C122757.

The activity of determining whether a test result value is within acceptable reference limits. 